\title{
Riscos da suplementação com doses excessivas de vitamina D
}

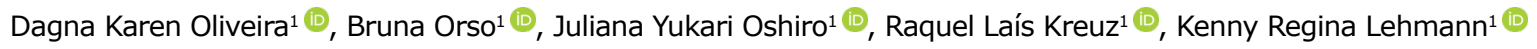

\begin{abstract}
RESUMO
A intoxicação por vitamina D era pouco frequente no Brasil até seu crescente uso na última década. Neste artigo relatamos um caso de intoxicação por vitamina $D$ em que houve a prescrição intencional de dose muito superior ao recomendado pela literatura, com a finalidade de prevenir doenças via "modulação hormonal". A paciente em questão, idosa, previamente hígida, foi submetida a um tratamento não regulamentado e sem respaldo científico, que culminou em sintomas como náuseas e vômitos, além de perda de peso, inapetência, poliúria e astenia ao longo dos meses. Através da história e exames laboratoriais foi diagnosticada intoxicação por vitamina $D$ e lesão renal aguda. Após o tratamento houve remissão completa dos sintomas. A "modulação hormonal" é uma prática condenada pelos Conselhos Federais de Medicina e Odontologia e pela Sociedade Brasileira de Endocrinologia e Metabologia. $O$ ato de prescrever é de grande responsabilidade ética e técnica e deve ser embasado em evidências científicas, oferecendo o melhor tratamento possível aos pacientes, seja ele preventivo ou curativo, minimizando riscos e danos, respeitando as recomendações das autoridades competentes.
\end{abstract}

Palavras-chave: Ética profissional, Geriatria, Lesão renal aguda, Toxicidade, Vitamina D.

1. Campus Toledo, Universidade Federal do Paraná (UFPR), Toledo (PR), Brasil 


\section{INTRODUÇÃO}

De importância singular ao sistema endócrino, a vitamina $D[25(\mathrm{OH}) \mathrm{D}]$ é um pró-hormônio, que atua como um regulador da fisiologia osteomineral, principalmente do metabolismo do cálcio e do equilíbrio de fósforo, além de intervir em outras funções fisiológicas, como a modulação da função muscular, do crescimento e da diferenciação celular. ${ }^{1,2}$

A intoxicação por vitamina $D$ era pouco frequente no Brasil até seu crescente uso na última década. ${ }^{3}$ Os diversos relatos na literatura destacam a intoxicação acidental, especialmente em idosos, devido a erro na ingesta, administração por cuidadores, falta de compreensão da prescrição ou erro na formulação. ${ }^{4,5}$

Neste artigo relatamos um caso de intoxicação no qual houve a prescrição intencional de uma dosagem muito acima do recomendado na literatura, com finalidade de prevenir doenças, com o subterfúgio de uma "modulação hormonal", prática condenada pelo Conselho Federal de Medicina (CFM), Sociedade Brasileira de Endocrinologia e Metabologia (SBEM) e Conselho Federal de Odontologia (CFO). ${ }^{6-8}$

\section{RELATO DO CASO}

Paciente do sexo feminino, 67 anos, procura serviço médico com náuseas, vômitos, constipação alternada com diarreia e fraqueza intensa, de início não preciso, com duração de três a quatro meses e piora progressiva. Além de poliúria, perda de peso, inapetência e insônia.

Nega comorbidades, história de cirurgias de catarata e varizes. Paciente se refere apenas uso de medicações prescritas por um profissional não médico para "modulação hormonal", preventivamente, pois estava envelhecendo e ambas as filhas fizeram tratamento recente para câncer (melanoma e câncer de mama). As medicações foram manipuladas e conforme as embalagens trazidas em consulta um dos preparados continha 50.000 UI Vitamina D por gota, e a orientação de utilizar 2 gotas diariamente.

Além de outro preparado contendo Vitamina A 4000 UI/dia, Vitamina K2 240 mcg/dia, Ocitocina sublingual $10 \mathrm{UI} /$ dia e Zinco $15 \mathrm{mg}$.

Realizava exercícios físicos há vários anos, suspendendo recentemente pela intensa astenia.
Ao exame físico, paciente de baixo peso, com IMC $18,7 \mathrm{~kg} / \mathrm{m}^{2}$, peso $44,5 \mathrm{~kg}$ e altura $1,54 \mathrm{~m}$, sem outras alterações.

Exames complementares realizados na semana anterior ao diagnóstico, solicitados por outro profissional, mostravam vitamina $\mathrm{D}$ acima de 210 $\mathrm{ng} / \mathrm{mL}$. Com suspeita de intoxicação por vitamina $D$, foi solicitada dosagem de cálcio, potássio e função renal para confirmação diagnóstica. Além de recomendação de hidratação e suspensão imediata do uso de vitamina $D$.

Com a confirmação diagnóstica da hipercalcemia (Cálcio total corrigido $12,9 \mathrm{mg} / \mathrm{dL}$ ) foi realizado internamento de urgência.

Durante internamento, realizada vigorosa hidratação endovenosa e estímulo de ingestão de água via oral, dieta restrita em cálcio. Após volemia adequada, iniciado o uso de furosemida e prednisona. Apresentou boa evolução clínica e laboratorial. Dois dias após o internamento recebeu alta hospitalar com prednisona por seis dias e seguimento ambulatorial.

Após cinco dias do diagnóstico, paciente apresentou melhora progressiva dos sintomas - fraqueza, constipação, apetite e boa evolução laboratorial (Quadro 1).

No seguimento ambulatorial realizou densitometria óssea, que mostrou osteoporose, sendo prescrito alendronato, via oral, doses semanais, para tratamento de tal condição e auxiliar na manutenção do cálcio sérico.

Quatro meses após cessar o uso dos suplementos vitamínicos, a paciente apresentava melhora total dos sintomas, recuperação do peso $(47,25 \mathrm{~kg})$ e retorno da rotina de exercícios físicos. Mantinha níveis elevados de Vitamina $D$.

Em concordância com a carta circular $n^{\circ}$ 166/2018-CONEP/SECNS/MS, esse relato de caso foi aprovado pelo CEP/SCS/UFPR parecer consubstanciado de número 4.161.100.

\section{DISCUSSÃO}

No caso relatado, a paciente foi em busca de tratamentos preventivos, pensando em seu envelhecimento e no fato de suas duas filhas recentemente terem sido diagnosticadas com formas diferentes e graves de câncer, sendo neste momento, atendida por profissional que a orientou realizar "modulação hormonal". 
Quadro 1 Resultados de exames por data. Toledo, PR, 2019/2020.

\begin{tabular}{|c|c|c|c|c|c|c|c|}
\hline & $\begin{array}{c}\text { Creatinina } \\
\text { (mg/dL) }\end{array}$ & $\begin{array}{c}\text { Cálcio Total } \\
\text { (mg/dL) }\end{array}$ & $\begin{array}{c}\text { Cálcio } \\
\text { Ionizado } \\
\text { (mmol/L) }\end{array}$ & $\begin{array}{c}\text { PTH } \\
(\mathrm{pg} / \mathrm{mL})\end{array}$ & $\begin{array}{c}\text { Vit D } 25(\mathrm{OH}) \\
\text { D } \\
(\mathrm{ng} / \mathrm{mL})\end{array}$ & $\begin{array}{c}\text { Ureia } \\
(\mathrm{mg} / \mathrm{dL})\end{array}$ & $\begin{array}{c}\text { Fósforo } \\
\text { (mg/dL) }\end{array}$ \\
\hline Referência & $0,5-1,0$ & $8,5-10,2$ & $1,05-1,30$ & $15-65$ & $30-60$ & $15-45$ & $2,5-4,8$ \\
\hline $\begin{array}{l}\text { Pré } \\
\text { Reposição }\end{array}$ & & 9,8 & & 69,4 & $*$ & & \\
\hline Diagnóstico & 1,37 & 13,4 & 1,59 & 28,4 & $>210$ & 66 & 4 \\
\hline Internamento & 0,89 & 9,02 & & & & 47,41 & 2,85 \\
\hline 4 dias** & 0,96 & 11 & 1,34 & & & 61 & \\
\hline 1 semana** & 0,88 & 10,6 & & & & 42 & \\
\hline 1 Mês** & 0,69 & 9,2 & 1,2 & & & 32 & \\
\hline 5 Meses** & 0,99 & & 1,2 & 57,7 & 89,03 & 48,11 & \\
\hline
\end{tabular}

Fonte: Laboratório, 2019/2020. *dosagem de 1,25 OH Vit D: 47,9 (ref 19,9 - 79,3) *^Após alta

A SBEM já emitiu parecer oficial esclarecendo que "(...) não existe especialista em Reposição Hormonal e não é reconhecida pelo CFM, especialidade intitulada "Modulação Hormonal". A reposição hormonal é um ato médico e somente pode ser feita por profissionais médicos. A especialidade que trata de alterações hormonais é a Endocrinologia e Metabologia. A reposição de qualquer hormônio deve ser feita baseada na deficiência dele, com acompanhamento médico especializado, observandose riscos e benefícios do uso; não há respaldo na literatura médica para uso de preparações hormonais com intuito de retardar o envelhecimento. (...)".7 Além da SBEM, outras entidades já se posicionaram contra tal prática, como o CFO, através da RESOLUÇÃO CFO-199, de 29 de janeiro de 2019, que cita: "(...) Ficam vedadas, ao cirurgião-dentista, a prescrição e a divulgação de terapias denominadas modulação e/ou reposição e/ou suplementação e/ou fisiologia hormonal, bem como a utilização de quaisquer outros termos não reconhecidos cientificamente, fora da sua área de competência e atuação". ${ }^{8}$

Além da modulação hormonal não ser uma prática aceita pelos conselhos de classe, a medicação prescrita a paciente continha o hormônio Ocitocina e doses elevadas de vitaminas com valores superiores aos recomendados pela ANVISA na RDC número 269/2005, que estabelece normas para níveis de dosagens diárias de vitaminas e minerais em medicamentos. ${ }^{9}$

A Resolução n. ${ }^{1}$ 1999/2012 do CFM regula e limita o uso de terapias hormonais com o objetivo de retardar, modular ou prevenir o processo de envelhecimento. Tal resolução também proíbe o uso de vitaminas e antioxidantes referidos como terapia antienvelhecimento, antiarteriosclerose, anticâncer ou direcionadas ao tratamento de doenças crônicodegenerativas; exceto em casos de deficiências com diagnóstico firmado, cuja reposição apresenta benefícios cientificamente comprovados. ${ }^{6}$

$\mathrm{Na}$ literatura ainda não há um consenso quanto ao papel da vitamina $D$ na prevenção de mortalidade, na redução do risco cardiovascular, câncer e doenças autoimunes. Os prováveis efeitos extra-esqueléticas da vitamina $D$ apresentam grande interesse científico, porém, até o momento, não há respaldo científico para a prescrição de suplementação de altas doses dessa substância objetivando efeitos além da saúde óssea. ${ }^{10-12}$

Entendemos, assim, que a prescrição realizada a paciente está em desacordo com a legislação e a literatura, uma vez que não há benefícios comprovados em relação à dosagem prescrita, mas sim, diversos riscos, agindo, portanto, o profissional com imperícia e imprudência. ${ }^{6,13}$

A apresentação da toxicidade da vitamina D pode variar de assintomática a sintomas graves, com risco de vida, em sua maioria decorrentes da hipercalcemia. ${ }^{12}$ Podem ser manifestações neuropsiquiátricas (confusão, psicose, estupor ou coma), cardiovasculares (complicações com o intervalo QT, elevação do segmento ST, bradiarritmias) ou gastrointestinais como no caso descrito: dor abdominal, náuseas, polidipsia, anorexia e constipação. ${ }^{5,14} \mathrm{~A}$ paciente apresentou também complicações renais: lesão renal aguda (clearance calculado 31,7 ) e desidratação.

O diagnóstico da intoxicação é firmado mediante a elevação das concentrações de $25(\mathrm{OH}) \mathrm{D}$ superior a 
$100 \mathrm{ng} / \mathrm{ml}$ seguida por hipercalcemia, hipercalciúria severas e por declínio ou indetectável atividade do hormônio da paratireoide (PTH). ${ }^{14}$

Neste caso, a paciente tinha a indicação para o rastreio de deficiência de vitamina $D$, com a dosagem da concentração de $25(\mathrm{OH}) \mathrm{D}$, pois se tratava de paciente idosa, com baixo peso, fatores de risco para osteoporose, que se confirmou durante a investigação. A suplementação com foco na saúde óssea só deveria ser realizada após confirmação de níveis abaixo do recomendado. ${ }^{15}$

A orientação em casos de insuficiência de vitamina D 25[OH]D (valores inferiores a $20 \mathrm{ng} / \mathrm{mL}$ ), é a suplementação de vitamina $D$ por meio de colecalciferol (D3) ou ergocalciferol (D2) com dose de ataque de $50.000 \mathrm{UI} /$ semana ou de $7.000 \mathrm{UI} /$ dia por oito semanas, sucedida da dose de suporte entre $1.000 \mathrm{e}$ $2.000 \mathrm{UI} / \mathrm{dia}^{5,16}$

A paciente recebeu a prescrição de uma dose de 700.000UI/semana, dose 14 vezes mais alta do que a dose de ataque, sem recomendação de tempo de uso, e sem dosagem prévia à prescrição.

A estratégia de tratamento da intoxicação por excesso de vitamina $D$ é composta por uma pausa da suplementação, redução da ingesta de cálcio, administração de solução isotônica de cloreto de sódio para restaurar a função renal e corrigir a desidratação, assim que o volume for restaurado e mantido, podem ser adicionados diuréticos de alça e glicocorticoides para ajudar a diminuir os níveis plasmáticos de cálcio, como realizados no caso relatado. ${ }^{14} \mathrm{Em}$ casos graves de hipercalcemia, a terapia antirreabsortiva com uso de calcitonina e bisfosfonatos pode ser útil. ${ }^{17-19}$

Para a redução da concentração de $25(\mathrm{OH})$ D no organismo, poderia ser utilizado fenobarbital, aminoquinolinas (cloroquina, hidrocloroquina), cetoconazol, rifampicina ou inibidores específicos de CYP27B1 (1a-hidroxilase). Todos os medicamentos com muitos efeitos colaterais indesejados e inúmeras interações medicamentosas, situações que devem ser evitadas, especialmente na população idosa. ${ }^{14}$

As consequências da suplementação com doses excessivas de vitamina $D$ são consideráveis, podendo levar a quadros de intoxicação com complicações severas, como lesões renais e desfechos potencialmente fatais. Tratamentos sem comprovações científicas, com promessas de juventude eterna e curas milagrosas são condenados pelas autoridades competentes. É válido destacar que a prescrição da substância, muitas vezes considerada inofensiva por alguns, culminou neste caso, em atendimentos especializados e internamento hospitalar gerando custos desnecessários ao sistema de saúde, uma vez que a paciente era previamente hígida. $\mathrm{O}$ ato de prescrever é de grande responsabilidade, o profissional deve se responsabilizar (ética e tecnicamente) não só pela prescrição, mas também pela assistência ao paciente durante todo o processo, realizando monitoramento dos desfechos e de efeitos adversos ou complicações, caso ocorram, realizando o pronto tratamento destes.

\section{REFERÊNCIAS BIBLIOGRÁFICAS}

1. Need AG, Morris HA, Horowitz M, Nordin C. Effects of skin thickness, age, body fat, and sunlight on serum 25-hydroxyvitamin D. Am J Clin Nutr. Dez 1993; 58(6): 882-5.

2. Amorim S S, Lima PJAT, Barroso IRDG, Ventura MM. Intoxicação por vitamina $D$ em paciente idosa: relato de caso. Geriatr Gerontol Aging. Mar 2019; 1(1): 1-4.

3. Lim Kenneth, Thadhani Ravi. Toxicidade da vitamina D. Braz. J. Nephrol. [Internet]. Junho de 2020 [citado em 13 de março de 2021]; 42 (2): 238-244. Disponível em: http:// www.scielo.br/scielo.php?script=sci_arttext\&pid=S010128002020000200238\&lng=en. Epub em 03 de abril de 2020. https://doi.org/10.1590/2175-8239-jbn-2019-0192.

4. Tebben PJ, Singh RJ, Kumar R. Vitamin D-Mediated Hypercalcemia: Mechanisms, Diagnosis, and Treatment. Endocrine Reviews. Out 2016; 37 (5): 521-47.

5. Taylor PN, Davies JS, A review of the growing risk of vitamin D toxicity from inappropriate practice. British Journal Of Clinical Pharmacological. Mar 2018; 84 (6): 1121-7.

6. Conselho Federal de Medicina. Resoluções Normativas no $1.999 / 2012$, de 19 de outubro de 2012. Dispõem sobre "A falta de evidências científicas de benefícios e os riscos e malefícios que trazem à saúde não permitem o uso de terapias hormonais com o objetivo de retardar, modular ou prevenir o processo de envelhecimento".

7. Endocrino.org.br [Internet]. Rio de Janeiro: Sociedade Brasileira de Endocrinologia e Metabologia; c2019 [acesso em 05 mai. 2020]. Disponível em: https://www. endocrino.org.br/alerta-sbem-nao-existe-especialistaem-modulacao-hormonal/

8. Conselho Federal de Odontologia. Resolução CFO n¹99, de 29 de janeiro de 2019. Dispõe sobre "Proíbe a realização de terapias denominadas de modulação e/ou reposição e/ou suplementação e/ou fisiologia hormonal por cirurgiões-dentistas fora de sua área de atuação, e dá outras providências". Disponível: http://transparencia. cfo.org.br/ato-normativo/?id=2919\&doing_wp_cron $=1$ 588475265.0936119556427001953125 
9. Agência Nacional de Vigilância Sanitária. Resolução RDC no 269, 22 setembro de 2005. Dispões sobre o "regulamento técnico sobre a ingestão diária recomendada de protéina, vitaminas e minerais". Disponível em: http://portal. anvisa.gov.br/documents/33916/394219/RDC_269_2005. pdf/2e95553c-a482-45c3-bdd1-f96162d607b3.

10. Endocrino.org.br [Internet]. Rio de Janeiro: Sociedade Brasileira de Endocrinologia e Metabologia; c2020 [acesso em 14 mar. 2021]. Disponível em: https://www. endocrino.org.br/nota-de-esclarecimento-vitamina-de-covid-19/

11. Maeda SS, Borba VZC, Camargo MBR, Silva DMW, Borges JLC, Bandeira $F$ et al. Recomendações da Sociedade Brasileira de Endocrinologia e Metabologia (SBEM) para o diagnóstico e tratamento da hipovitaminose D. Arq Bras Endocrinol Metab. Jul 2014; 58 (5): 411-33.

12. Catarino AM, Claro C, Viana I. Vitamina D - Perspectivas Atuais. Revista SPDV. 21 Nov 2016; 74 (4): 345-53.

13. Oliveira TFL, Oliveira LSAF, Santos L, Mascarenhas C, Lopes N, Dantas P. Responsabilidade civil em odontologia - uma visão por profissionais da área jurídica. Odontol. Clín.-Cient. Out./Dez. 2013; 12 (4): 261-4.

14. Marcinowska-Suchowierska E, Kupisz-Urbańska M, Łukaszkiewicz J, Pludowski P, Jones G. Vitamin D Toxicity-A
Clinical Perspective. Frontiers in Endocrinology. Set 2018; 9 (550): 1-7.

15.Pludowski P, Holick MF, Grant WB, Konstantynowicz J, Mascarenhas MR, Haq A et al. Vitamin D supplementation guidelines. The Journal of Steroid Biochemistry and Molecular Biology. Jan 2018; 175 (1): 125-35.

16. Radominski SC, Bernardo $\mathrm{W}$, Paula $\mathrm{AP}$, Albergaria $\mathrm{BH}$, Moreira C, Fernandes CE et al. Brazilian guidelines for the diagnosis and treatment of postmenopausal osteoporosis. Rev Bras Reumat. Mai 2017; 57 (2): 452-66.

17. Diniz LR, Garcia JMA, Peixoto RI, Diniz KLO, Monteiro VS. Filtração glomerular em idosos coronarianos agudos: cockcroft-gault é o melhor método? Geriatr Gerontol 9Aging. Jan 2015; 9 (1): 8-13.

18. Marques MA, Judas FJM, Dias RMF. Indicações e Complicações dos Bifosfonatos em Ortopedia. Artigo de revisão. (dissertação). Faculdade de Medicina da Universidade de Coimbra; 2014.

19. Shih AWY, Weir MA, Clemens KK, Yao Z, Gomes T, Mamdani MM et al. Oral bisphosphonate use in the elderly is not associated with acute kidney injury. Official Journal Of The International Society Of Nephrology. Out 2012; 82 (8): 903-8. 


\section{Conflito de interesse}

Os autores declaram não haver conflito de interesse.

Financiamento Próprio.

Autor Correspondente:

Dagna Karen Oliveira

dagnaoliveira2015@gmail.com

Editor:

Prof. Dr. Paulo Henrique Manso

Recebido: 03/09/2020

Aprovado: 05/05/2021 